\title{
Diferencias entre universidades privadas y oficiales venezolanas: Algunos ajustes necesarios en el sector privado
}

\author{
Fuenmayor Toro, Luis*
}

\section{Resumen}

Este trabajo efectúa una caracterización preliminar de la universidad privada venezolana, a medio siglo de sus inicios. Se determinan algunas de sus fallas principales y propone correctivos factibles aprovechando las leyes de educación próximas a ser aprobadas. Los datos demuestran que las universidades privadas, a diferencia de las oficiales, tiene una planta docente mayoritariamente a tiempo convencional, ubicada principalmente en los más bajos escalafones académicos, con estur dios de postgrado en una proporción menor que las universidades oficiales y prácticamente sin investigadores. La relación alumno/profesor es el doble de la del sector oficial y los postgrados universitarios que ofrecen son la quinta parte del total y sólo están acreditados el 10\%, en contraposición con el $29 \%$ acreditado de las instituciones públicas. De estas comparaciones se extrae que la universidad privada está muy limitada para la realización de investigación y para el desarrollo de los postgrados que forman investigadores: maestrías y doctorados. Tiene además limitaciones subsanables en el pregrado, por no disponer de docentes contratados a altas dedicaciones, que dispongan de tiempo dentro de su dedicación para las consultas estudiantiles. Esto se puede resolver a través de un mandato legal que determine que un $33 \%$ de los docentes, sin contar autoridades y directivos, deban estar a dedicación exclusiva y repartidos homogéneamente entre todos los programas docentes institucionales. Estos docentes, también por mandato legal, dispondrían de un $30 \%$ de su tiempo para hacer investigación en unidades de universidades y otros centros oficiales, a las que serían adscritos formalmente. Ello mejoraría la academia de las instituciones privadas sin costos que hagan imposible estos importantes cambios.

Palabras clave: Tipos de universidades, universidades privadas, universidades oficiales, educación superior, indicadores de desempeño universitario.

Recibido: 03-03-14 . Aceptado: 03-05-16

Médico. Profesor Titular, Jubilado. Ex Rector de la U.C.V. Investigador en el área de Educación Superior. Director de la Oficina de Planificación del Sector Universitario (OPSU).

E-mail: sidalj@hotmail.com 


\section{Differences Between Private and Governmental Venezuelan Universities: Necessary Adjustments in the Private Sector}

\section{Abstract}

This paper presents a preliminary characterization of Venezuelan private universities half a century after their founding. Some of their principal faults are determined and feasible correctives are proposed in relation to educational laws that are soon to be passed. The information indicates that private universities, in contrast with public universities, have an academic staff working part-time, most of which are classified on the lower levels of the academic hierarchy scale, and with a lower percentage of post-graduate studies in comparison with professors in official public universities. The number of researchers is practically nil in private universities. In private universities the number of students for each professor is double that of public universities, and the postgraduate programs offered are onefifth the number of public universities. Only $10 \%$ of private universities are accredited as opposed to $29 \%$ of public universities. Based on these comparisons the private university is very limited in the area of research and the development of post-graduate programs that could form researchers (Masters and Doctoral degrees). Private universities also have unsolvable limitations in their undergraduate programs since they do not have professors who can dedicate time to students advising. This could be resolved by a legal mandate that stipulated that $33 \%$ of professors should be contracted under the figure of "total dedication" (not including university authorities) and distributed equitable among the different degree areas. These professors could dedicate $30 \%$ of their time to research in university programs or in official governmental programs and offices, to which they would be officially assigned. All of this would considerably improve these private institutions without generating excessive costs which would hurt their functioning.

Key words: Types of universities, private universities, official universities, higher education, higher education efficiency indicators.

\section{Introducción}

La historia de la educación superior privada en Venezuela tiene apenas medio siglo de duración, ya que se inicia en el año 1953, durante el gobierno del General Marcos Pérez Jiménez, con la fundación de dos instituciones universitarias: la Universidad Santa María el 13 de octubre y la Universidad Católica Andrés Bello, 6 días después, el 19 de octubre (Fuenmayor, 2002:3). La primera es fundada por los educadores Lola Rodríguez de Fuenmayor y Asdrúbal Fuenmayor Ri- vera, quienes ya poseían una institución de educación primaria y secundaria en el país, el Colegio Santa María, una de las pocas instituciones educativas privadas venezolanas que no estaba en manos de religiosos para aquel momento y que, junto con la universidad creada, le permitió refugio dentro de su planta docente a profesores perseguidos por el régimen de Pérez Jiménez. La Andrés Bello, por su parte, fue fundada por la Compañía de Jesús, la cual añadió a su ya importante presencia educativa en Venezuela su primera universidad. 
Debieron pasar casi doce años, para que apareciera una nueva universidad privada: la Universidad Metropolitana el 24 de febrero de 1965 (Fuenmayor, 2002:3) pues las condiciones económicas, sociales y educativas de la Venezuela pérezjimenista escasamente permitían la existencia de muy pocas universidades: tres oficiales y dos privadas, suficientes para garantizar la formación de los profesionales que requería la actividad económica y social existente, el pequeño aparato burocrático del Estado y las necesidades personales de servicio de la burguesía venezolana. Es sólo a partir de finales de los años setenta, -a medida que las necesidades creadas por la extensión de la política de sustitución de importaciones a los bienes intermedios y de capital presionan al sistema educativo-, que realmente se inicia una rápida expansión de la actividad educativa privada en el nivel universitario, en respuesta a las necesidades de formación de la alta gerencia privada (Fuenmayor, 1986:2) y, además, como una inversión segura, de gran reconocimiento social y, sobretodo, muy rentable.

La Universidad Metropolitana es una institución fundada por la gran burguesía venezolana, como una forma de participar directamente en la formación de sus tablas gerenciales y productivos y de dirigir la enseñanza universitaria hacia aquellas carreras y programas fuertemente vinculados con la etapa de desarrollo de la producción capitalista venezolana del momento. Se trataba del modelo a ser copiado por los futuros inversionistas en el campo educativo universitario, modelo que no era compartido en ese entonces por la Universidad San- ta María, que venía curiosamente desarrollando un modelo populista de universidad privada (Ágreda, 1986), si es que esta categoría realmente existe; tampoco por la Universidad Católica Andrés Bello, que respondía más al interés de continuar con estudios de alto nivel, la formación de aquellos ciudadanos iniciada en los colegios católicos del país, con el objetivo de prepararlos y formarlos para asumir el gobierno y administración de la nación en sus distintos niveles e instancias regionales.

Comienza entonces a configurarse una tabla educativa en el sector privado universitario, que responde a una mezcla de intereses y que, por lo tanto, no llega a satisfacer enteramente las necesidades de expansión de la producción capitalista venezolana. Esta heterogeneidad de intereses lleva a las instituciones privadas a configurar también un sistema heterogéneo de educación universitaria, con características académicas particulares, distintos a las existentes en las universidades oficiales y con diferentes niveles de calidad, incluso dentro de una misma institución, lo cual constituye el principal problema a tratar de resolver en el futuro cercano, aprovechando para ello el establecimiento del Sistema de Evaluación y Acreditación (SEA) de las Universidades Nacionales, ya aprobado por el Consejo Nacional de Universidades (CNU) a proposición de la Oficina de Planificación del Sector Universitario (OPSU), así como la próxima promulgación de la Ley Orgánica de Educación y, un poco después, de las leyes de educación superior. Caracterizar adecuadamente a la universidad privada venezolana se hace pues importante y perentorio, si se quiere producir los 
ajustes que mejoren su utilidad en el enfrentamiento de las necesidades del país y de los múltiples problemas que aquejan a la sociedad venezolana.

Lo afirmado anteriormente no significa que estamos en presencia de una universidad privada de desarrollo completamente diferente al de la universidad oficial. De hecho, ambas existen y trabajan en una mismo país, con limitaciones y retos comunes, sujetas a los vaivenes económicos y políticos nacionales, todo lo cual tendrá también que ser abordado, para corregir lo procedente de la mejor manera posible, siempre en función del interés colectivo de la patria.

La universidad oficial también constituye un grupo heterogéneo, no sólo en aquellas características en las cuales es lógica y pertinente la diferencia, dada la orientación con la que fueron creadas y las necesidades particulares que satisfacen; sino también en aspectos de equidad, eficiencia, pertinencia social, eficacia, impacto y desarrollo integral de las funciones que le son propias: docencia, investigación y extensión. Estas diferencias han sido recientemente demostradas en forma clara en un estudio realizado por el "Proyecto Alma Mater para el mejoramiento de la calidad y equidad de la educación universitaria en Venezuela", auspiciado por la OPSU; y permiten hablar sobre bases sólidas de la existencia de varios tipos de universidades oficiales venezolanas, pues las grandes diferencias existentes entre las mismas permiten incluso clasificarlas en grupos claramente distintos (CNU-OPSU, 2002). Dentro de esta diversidad, la calidad se transforma en el problema principal a enfrentar y resolver, pues en ella se integran todas las cualidades requeridas por este nivel educativo.

\section{La calidad como problema fundamental en docencia, investigación y extensión}

A la hora de caracterizar una institución educativa, estudiar la calidad de la misma se convierte en una tarea imprescindible y urgente. En Venezuela, tanto el sistema educativo como el de salud son mixtos en lo que se refiere a la prestación de servicios, lo que quiere decir que la misma es asumida tanto por el sector oficial como por el sector privado. Independientemente de las posiciones ideológicas que se tengan al respecto, la prestación mixta de los servicios es una realidad, por lo cual los estudios y observaciones que se pudieren hacer sobre las características de ambos sistemas deben tomar en consideración esa premisa fundamental. En el caso de la prestación de los servicios educativos, no está planteada en los momentos actuales la desaparición de la prestación privada de los mismos. A pesar de lo anterior, también es claro que constitucionalmente es el Estado el responsable de ambos servicios, lo que constituye una función esencial y una obligación del mismo.

En el caso que nos compete, para asumir el cumplimiento de funciones educativas universitarias, el Estado delega parte de la prestación de los servicios en el sector privado, pero se reserva la supervisión permanente, el dictado de normas, la evaluación y el control de los mismos a través de las leyes sobre la materia; estas le permiten revertir el permiso otorgado a una institución, en caso de in- 
cumplimiento por parte de cualquier particular que se encuentre prestando servicios, tal y como lo establece el artículo 183 de la Ley de Universidades vigente.

Para el Estado, como ya lo hemos dicho, lo fundamental en un sistema de prestación mixta de servicios es la calidad con la cual esos servicios se presten. Sobretodo en servicios de las características de los señalados, en los que la atención tiene que ser de buena calidad; no se pueden aceptar, independientemente de que existan y en proporción importante, servicios de regular o de mala calidad. Así, el artículo 103 de la Constitución Nacional venezolana establece que toda persona tiene derecho a una "educación integral, de calidad, permanente, en igualdad de condiciones y oportunidades", lo cual significa que el Estado venezolano está en la obligación de asegurar este derecho constitucional, cualquiera que sea el prestador de los servicios educativos: el sector oficial o el sector privado.

Con relación a la definición de "calidad universitaria" hacemos nuestra la esbozada en el seminario sobre educación superior en el siglo XXI, realizado en La Habana en 1996 (UNESCO, 1997) que la concibió como "la adecuación del ser y quehacer de la educación superior a su deber ser. El "ser" se refiere a lo que es la institución en el presente, sus resultados y logros actuales, que pueden ser evaluados en términos de efectividad y eficacia; el "quehacer" es el papel del funcionamiento institucional, el cual puede ser medido en términos de eficiencia, y el "deber ser" define y caracteriza a la institución, sus objetivos, planes y proyectos, y es evaluado en términos de pertinencia
(OPSU, 2001:21). Esta descomposición del concepto de calidad en sus categorías evaluativas permite la elaboración de índices para medir la calidad en general y para las distintas categorías mencionadas (CNU-OPSU, 2001:23), lo que facilita su medición, hace muy objetivo el proceso y permite una mejor comparación entre instituciones diferentes. La medición de la calidad, por supuesto, tiene que ir dirigida, aunque no exclusivamente, hacia las actividades esenciales universitarias: docencia (pregrado y postgrado), investigación y extensión.

Si en algo hay que caracterizar a la universidad privada venezolana, como sistema heterogéneo y con distintas motivaciones, es con relación al desarrollo de sus actividades esenciales: docencia, investigación y extensión. Pero de todas ellas, como demostraremos más adelante, y a diferencia de la universidad oficial, es la docencia de pregrado la actividad fundamental, casi exclusiva, de las universidades privadas y es ésta la que debe ser evaluada preferentemente, con criterios bastante similares a los utilizados en la evaluación de las universidades oficiales.

Con esto quiero señalar, y es preciso que se entienda con sentido de realidad, que si en Venezuela se acepta la existencia y funcionamiento del sector privado en la educación universitaria, no es por necesidades de producción de conocimientos ni de desarrollo general de los postgrados, ni de la realización de actividades de extensión. Es, casi exclusivamente, por la necesidad de atención de una matrícula de pregrado creciente que el Estado no está, por el momento, en capacidad de atender. Es esta necesidad la 
que debe dictar la pauta en la caracterización de las universidades privadas, ya que el postgrado, la investigación y la extensión, deberían estar circunscritas a las reales posibilidades de estas instituciones de llevarlas a cabo en forma adecuada o al menos similar a como lo realizan las universidades oficiales.

Es imposible exigirle a las universidades privadas venezolanas la realización de investigación científica de gran complejidad y en la misma magnitud como se le debe exigir a la universidad oficial. Las labores de investigación son muy costosas y el financiamiento de las universidades privadas venezolanas no permitiría sostener el desarrollo de este tipo de actividades con la complejidad y en la magnitud en que pueden ser realizadas por las universidades oficiales. El pago de matrícula por parte de los estudiantes de pregrado, principal y muchas veces fuente única de financiamiento, se elevaría a montos inaccesibles si se efectuara una exigencia de este tipo. Esto, por supuesto, no significa que no se deban efectuar exigencias, incluso nuevas, en la materia. Las mismas deben producirse. Pero esas exigencias deberían ser factibles, pues si no lo son quedarían como letra muerta en leyes, reglamentos y normas, como ha sido el caso hasta ahora. Esto significa que en las exigencias que se pretendan instrumentar se deben reunir tres requisitos: ser cumplibles por las universidades privadas, se debe estar en capacidad de hacer obligatorio su cumplimiento y éste debe beneficiar a la institución, al docente investigador y al país.

Con relación a los postgrados, este trabajo de investigación pretende contribuir a sincerar la situación existente, pues la misma ha sido desdibujada y motivada casi exclusivamente por un interés mercantilista, que ha obligado incluso al Consejo Nacional de Universidades a introducir una serie de correctivos. Se pretende entonces definir algunas líneas de acción o ideas que permitan conducir a un procedimiento que defina el tipo y las características de los postgrados cuyo dictado pueda ser permitido a las instituciones privadas; esto tomando en consideración sus naturales limitaciones en nuestros países y una vez cubiertos los requisitos que aseguren la calidad en el dictado de los programas de que se trate. Esto no significa ni debe entenderse como que existen postgrados cuyo dictado está vedado a las universidades privadas. No. Significa que en caso de postgrados de difícil ejecución por lo costosos, el tipo de infraestructura necesaria y la necesidad de personal académico de muy elevado nivel, el estudio para su aprobación debe ser lo más riguroso y exigente posible y su supervisión y evaluación deben ser permanentes en aras de garantizar su calidad.

La extensión no será analizada, pues existe poca información disponible al respecto, incluso de las instituciones oficiales, ya que es la actividad esencial universitaria de menor desarrollo. Sin embargo, algunas ideas serán expuestas en orden de garantizar su desarrollo en el futuro, sobretodo en lo que respecta a la relación extensión/docencia y pregrado/prestación de servicios.

Esta investigación nos permitirá profundizar más en el conocimiento de la realidad universitaria del país, concretamente con relación a la universidad privada venezolana, cuya caracterización no 
ha sido bien explorada. También nos permitirá sugerir adiciones en la legislación educativa superior con miras a prevenir distorsiones en sus actividades, corregir situaciones inconvenientes, mejorar el funcionamiento institucional e incrementar su utilidad social; en síntesis, mejorar la calidad de la universidad privada venezolana.

\section{Metodología}

Se trabajará con información cuantitativa y con aquellos indicadores con los cuales usualmente se describen, se miden y se comparan las actividades académicas y administrativas de las universidades, tanto públicas como privadas. Se trata del mismo tipo de datos o indicadores utilizados por el "Sistema de Evaluación y Acreditación" de la OPSU, coordinado por el proyecto "Alma Mater" (OPSU, 2001). Estas cifras e indicadores tienen la ventaja de que fueron discutidos por todas las universidades, oficiales y privadas, durante el año 2001 y tienen el consenso de las mismas.

Los datos cuantitativos e indicadores escogidos se presentarán para cada una de las 17 universidades privadas que existían para el año 1999: Santa María (USM), Católica Andrés Bello (UCAB), Metropolitana (UNIMET), Rafael Urdaneta (URU), Tecnológica del Centro (UNITEC), Católica del Táchira (UCAT), José María Vargas (UJMV), Cecilio Acosta (UNICA), Bicentenaria de Aragua (UBA), Gran Mariscal de Ayacucho (UGMA), Fermín Toro (UFT), Nueva Esparta (UNE), Yacambú (UNY), Rafael Belloso Chacín (URBE), Valle del Momboy (UVM), Alejandro de Humboldt (UAH) y
José Antonio Páez (UJAP). Se decidió descartar, al igual que se hizo con las universidades oficiales, aquellas instituciones que no alcanzaran los cinco años de fundadas o de haber iniciado sus actividades académicas, pues las mismas no han completado aún el dictado de los programas propuestos de pregrado del nivel de licenciatura y, por lo tanto, tienen una matrícula estudiantil menor que la calculada para todas las carreras y un número de profesores por debajo de los necesarios para el dictado de todos los cursos inicialmente propuestos. Se trata de universidades "recién nacidas", que aún están en la etapa inicial de su crecimiento y desarrollando los programas y carreras propuestos originalmente para su creación. Además, posiblemente sin concluir la planta física mínima necesaria para su funcionamiento.

Los datos serán comparados con los existentes en las universidades oficiales, las cuales serán divididas en este estudio en dos grandes grupos: un grupo estará constituido por las 5 universidades autónomas: Central de Venezuela (UCV), Los Andes (ULA), Zulia (LUZ), Carabobo (UC) y Oriente (UDO), supuestamente las de mayor desarrollo, a las que se añadió la Universidad Simón Bolívar (USB), universidad de carácter experimental, ya que todas ellas han sido consideradas como pertenecientes al mismo tipo de universidades (OPSU, 2002) por presentar grandes similitudes en sus retos $y$ actividades.

El otro grupo de universidades oficiales está constituido por las 11 universidades experimentales restantes que existían también para 1999, supuestamente de menor desarrollo: Centrocci- 
dental Lisandro Alvarado (UCLA), Nacional Experimental Simón Rodríguez (UNESR), Nacional Experimental del Táchira (UNET), Nacional Experimental de los Llanos Occidentales Ezequiel Zamora (UNELLEZ), Nacional Abierta (UNA), Nacional Experimental Rómulo Gallegos (UNERG), Nacional Experimental Francisco de Miranda (UNEFM), Nacional Experimental de Guayana (UNEG), Nacional Experimental Rafael María Baralt (UNERMB), Pedagógica Experimental Libertador (UPEL), Nacional Experimental Politécnica Antonio José de Sucre (UNEXPO). En este caso se descartó también a 4 instituciones de reciente creación, con menos de 5 años de vida académica, por las mismas razones aducidas anteriormente para las universidades privadas. Sin embargo, las universidades Nacional Experimental de la Fuerza Armada y Nacional Experimental Marítima del Caribe fueron incluidas en la parte de esta investigación correspondiente al postgrado, pues algunas de las instituciones que formaron parte de su constitución tenían un desarrollo previo de postgrado.

Se presentarán los datos individuales de cada institución, en cifras absolutas y porcentuales, así como el promedio de cada uno de los indicadores para cada grupo descrito de universidades. La calidad de las condiciones académicas generales y de la docencia de pregrado será evaluada mediante los siguientes indicadores: Número y proporción de profesores por dedicación, número y proporción de docentes por categoría (escalafón) y; número de alumnos por profesor "tiempo completo equivalente". El número total de profesores tiempo completo equivalente se obtuvo sumando el número de profesores a tiempo completo más la mitad de los profesores a medio tiempo más la cuarta parte de los profesores a tiempo convencional (OPSU, 2001: 40).

La preparación y formación del personal docente, la cual repercute en toda la actividad académica, se evalúa mediante la aplicación de los siguientes indicadores: Número total de profesores con título de postgrado; proporción de profesores activos con título de postgrado, número total de profesores reconocidos por el Programa de Promoción del Investigador (PPI) y la proporción de profesores a dedicación exclusiva + tiempo completo reconocidos por el PPI.

Se evaluará también el número de postgrados activos y el tipo de los mismos: especializaciones, maestrías y doctorados, así como el de los postgrados acreditados, en cada uno de los grupos de universidades señalados, para efectuar las comparaciones correspondientes y proceder al análisis de las diferencias.

Se seleccionó la modalidad de investigación documental, la cual es una variante de la investigación científica, cuyo objeto es el estudio de diferentes fenómenos de la realidad, a través de la indagación exhaustiva, sistemática y rigurosa, de la documentación existente, que aporte información atinente al fenómeno que se estudia (Ramírez, Méndez y Bravo, 1988: 21). Esta investigación es del tipo analítico-descriptivo-interpretativo, el cual se refiere a los estudios "cuyo alcance se extiende hasta la determinación de la frecuencia con que algo ocurre o con la que se halla asociado o relacionado con otro factor" (Selltiz y Jahoda, 1977: 22). 


\section{Resultados}

\subsection{Dedicación a la Universidad de los Profesores Activos}

El número y proporción de profesores clasificados por tiempo de dedicación de las universidades privadas venezolanas se presenta en la Tabla 1. Las cifras demuestran claramente que la inmensa mayoría de los docentes se encuentran contratados a tiempo convencional, es decir por horas de dictado de clases. Este grupo constituye más de las tres cuartas partes de todos los profesores, aunque en más de un tercio de las instituciones la proporción casi alcanza o supera el $90 \%$ del total de docentes. Los contratados a dedicación exclusiva, en cambio, son menos del dos por ciento del total de profesores y en el $64,7 \%$ de las instituciones no existen docentes de esta dedicación.

Los contratados a tiempo completo están, en promedio, ligeramente por encima del 10 por ciento. Individualmente, sin embargo, existen unas pocas instituciones, cinco en total, que tienen profesores contratados a tiempo completo en una proporción igual o mayor del 20 por ciento; dos de estas cinco universidades tienen contratados en esta dedicación a más del 30 por ciento de sus docentes. Se trata en casi todos los casos, sin embargo, de aquellas instituciones que no tienen personal a dedicación exclusiva. Las tres universidades privadas de más reciente creación, excluidas de este estudio, tienen un patrón de contratación de profesores exactamente similar al descrito (datos no mostrados).
En el grupo (experimental) de las universidades oficiales, que incluye a las autónomas y a la Universidad Simón Bolívar, el número y proporción de los profesores contratados a diferentes dedicaciones sigue un patrón completamente inverso (Tabla 2) al descrito para las universidades privadas. La mitad de los profesores está contratado a dedicación exclusiva, la máxima posible, y casi un $30 \%$ a tiempo completo, mientras que los profesores contratados a medio tiempo constituyen una proporción ligeramente superior al $10 \%$ del total y los docentes contratados por horas son sólo la quinta parte del total de profesores existentes en este grupo de instituciones.

Dos universidades se destacan por tener cifras elevadas de contratación de docentes a dedicación exclusiva (Tabla 2); una de ellas, la Universidad de Los Andes, con casi las tres cuartas partes de sus profesores a esa dedicación; lo hace a expensas de los docentes contratados a tiempo completo y a medio tiempo, mientras que la otra, la Universidad de Oriente, lo hace a expensas de los profesores contratados a tiempo convencional, los cuales en dicha institución constituyen el $6 \%$ aproximadamente, cifra bastante inferior al promedio de docentes contratados a esta dedicación en el grupo de universidades oficiales autónomas.

Es importante señalar que del total de profesores de este grupo de universidades oficiales (16.225) más de la cuarta parte (4.828) pertenece a la Universidad Central de Venezuela en todas las categorías. 


\section{Tabla 1}

Dedicación de los profesores activos de las universidades privadas venezolanas en el año 2000

\begin{tabular}{lrrrrrrrr}
\hline & \multicolumn{2}{c}{$\begin{array}{c}\text { Dedicación } \\
\text { Exclusiva }\end{array}$} & \multicolumn{2}{c}{$\begin{array}{c}\text { Tiempo } \\
\text { Completo }\end{array}$} & \multicolumn{2}{c}{$\begin{array}{c}\text { Medio } \\
\text { Tiempo }\end{array}$} & \multicolumn{2}{c}{ Tiempo } \\
Convencional
\end{tabular}

Fuente: OPSU (2001).

En la Tabla 3 se presentan los datos de la dedicación de los profesores activos del grupo correspondiente a las universidades oficiales experimentales sin la Universidad Simón Bolívar. El patrón de dedicación resultante es muy parecido al del grupo de las universidades autónomas y la Simón Bolívar (Tabla 2), pues el mayor porcentaje de profesores se encuentra contratado en las dedicaciones mayores a la universidad: dedicación exclusiva y tiempo completo, las cuales incorporan a más del $60 \%$ del total de profesores. Otro tanto ocurre con la proporción de los docentes contratados a medio tiempo (Tabla 3), la cual constituye alrededor de la décima parte del total de profesores activos y es muy similar a la del otro grupos de universidades oficiales (Tabla 2), el doble de la proporción mos- 


\section{Tabla 2}

Dedicación de los profesores activos del grupo de seis universidades oficiales venezolanas integrado por las cinco universidades autónomas y la Universidad Simón Bolívar en el año 2000

\begin{tabular}{lcccrrrrr}
\hline & \multicolumn{2}{c}{$\begin{array}{c}\text { Dedicación } \\
\text { Exclusiva }\end{array}$} & \multicolumn{2}{c}{$\begin{array}{c}\text { Tiempo } \\
\text { Completo }\end{array}$} & \multicolumn{2}{c}{$\begin{array}{c}\text { Medio } \\
\text { Tiempo }\end{array}$} & \multicolumn{2}{c}{$\begin{array}{c}\text { Tiempo } \\
\text { Convencional }\end{array}$} \\
\hline Universidades & $\mathbf{N}^{\circ}$ & \multicolumn{1}{c}{$\%$} & \multicolumn{1}{c}{$\mathbf{N}^{\circ}$} & \multicolumn{1}{c}{$\%$} & $\mathbf{N}^{\circ}$ & $\%$ & $\mathbf{N}^{\circ}$ & $\%$ \\
\hline UCV & 1.841 & 38,13 & 943 & 19,53 & 863 & 17,87 & 1.181 & 24,46 \\
ULA & 1.984 & 72,70 & 260 & 9,53 & 45 & 1,67 & 440 & 16,12 \\
LUZ & 1.498 & 49,28 & 869 & 28,59 & 312 & 10,26 & 361 & 11,88 \\
UC & 817 & 36,95 & 258 & 11,67 & 347 & 15,69 & 789 & 35,69 \\
UDO & 1.389 & 62,09 & 528 & 23,60 & 186 & 8,31 & 134 & 5,99 \\
USB & 511 & 43,31 & 220 & 18,64 & 0 & 0,00 & 449 & 38,05 \\
Total & 8.040 & 49,55 & 3.078 & 18,97 & 1.753 & 10,80 & 3.354 & 20,67 \\
\hline
\end{tabular}

Fuente: OPSU (2001).

\section{Tabla 3}

Dedicación de los profesores activos del grupo de once universidades oficiales venezolanas experimentales en el año 2000

\begin{tabular}{lccrrrrrr}
\hline & \multicolumn{2}{c}{$\begin{array}{c}\text { Dedicación } \\
\text { Exclusiva }\end{array}$} & \multicolumn{2}{c}{$\begin{array}{c}\text { Tiempo } \\
\text { Completo }\end{array}$} & \multicolumn{2}{c}{$\begin{array}{c}\text { Medio } \\
\text { Tiempo }\end{array}$} & \multicolumn{2}{c}{$\begin{array}{c}\text { Tiempo } \\
\text { Convencional }\end{array}$} \\
\hline Universidades & \multicolumn{1}{c}{$\mathbf{N}^{\circ}$} & \multicolumn{1}{c}{$\%$} & \multicolumn{1}{c}{$\mathbf{N}^{\circ}$} & \multicolumn{1}{c}{$\%$} & $\mathbf{N}^{\circ}$ & $\%$ & $\mathbf{N}^{\circ}$ & $\%$ \\
\hline UCLA & 305 & 24,98 & 513 & 42,01 & 159 & 13,02 & 244 & 19,98 \\
UNESR & 495 & 37,93 & 231 & 17,70 & 135 & 10,34 & 444 & 34,02 \\
UNET & 152 & 46,06 & 132 & 40,00 & 25 & 7,58 & 21 & 6,36 \\
UNELLEZ & 422 & 60,11 & 1 & 0,14 & 15 & 2,14 & 264 & 37,61 \\
UNA & 113 & 20,81 & 115 & 21,18 & 285 & 52,49 & 30 & 5,52 \\
UNERG & 134 & 23,63 & 64 & 11,29 & 10 & 1,76 & 359 & 63,32 \\
UNEFM & 283 & 40,60 & 210 & 30,13 & 34 & 4,88 & 170 & 24,39 \\
UNEG & 116 & 36,94 & 136 & 43,31 & 48 & 15,29 & 14 & 4,46 \\
UNERMB & 396 & 83,37 & 44 & 9,26 & 10 & 2,11 & 25 & 5,26 \\
UPEL & 661 & 20,13 & 511 & 15,57 & 510 & 15,53 & 1.601 & 48,77 \\
UNEXPO & 472 & 68,01 & 121 & 17,44 & 75 & 10,81 & 26 & 3,75 \\
Total & 3.549 & 42,05 & 2.078 & 22,54 & 1.306 & 12,35 & 3.198 & 23,04 \\
\hline FU, & & & & & & &
\end{tabular}

Fuente: OPSU (2001). 
trada por las universidades privadas (Tabla 1). Los docentes contratados a tiempo convencional, es decir aquéllos que trabajan por horas efectivas de docencia dictadas, constituyen una proporción similar al del otro grupo de universidades oficiales.

También en este grupo de universidades oficiales existen algunas con unos porcentajes de profesores a dedicación exclusiva elevados con relación al promedio del grupo (Tabla 3). En el caso de la UNELLEZ esta elevada cifra se tiene a expensas de los profesores contratados a tiempo completo, prácticamente inexistentes en dicha institución, y de aquéllos a medio tiempo. En el caso de la Rafael María Baralt, la elevada proporción docente a dedicación exclusiva se mantiene a expensas del resto de las contrataciones de menor dedicación, todas ellas en proporciones muy por debajo del promedio del grupo. En la Universidad Politécnica el elevado número a dedicación exclusiva es a expensas de la muy baja contratación de profesores a tiempo convencional (Tabla 3).

En los casos de la UCLA, la UNET y la Universidad de Guayana, la mayor proporción de su personal está contratado a tiempo completo, mientras que las universidades Rómulo Gallegos y la Pedagógica Libertador tienen la mayor parte de sus docentes contratados a tiempo convencional (Tabla 3). Esta última tendencia está presente en las cuatro universidades oficiales experimentales no incluidas en el presente estudio por ser de muy reciente creación (datos no mostrados).

\subsection{Categoría en el escalafón docente de los profesores activos}

Los profesores clasificados por categoría (escalafón) de los tres grupos de universidades estudiados en el presente trabajo se muestran en las Tablas 4, 5 y 6 , los cuales reflejan las cifras absolutas y porcentuales del número de docentes de cada nivel del escalafón y los totales de cada grupo: universidades privadas (Tabla 4), universidades oficiales autónomas más la Universidad Simón Bolívar (Tabla 5) y universidades oficiales experimentales sin la Simón Bolívar (Tabla 6). De la comparación de los tablas mencionados se puede apreciar que las universidades privadas concentran casi el 80 por ciento de sus docentes en las más bajas categorías: las de instructor y asistente, mientras los docentes titulares sumados a los del nivel de asociado, constituyen menos del 10 por ciento del escalafón docente del nivel universitario privado.

En el grupo de las universidades autónomas y la Simón Bolívar, en cambio, los profesores instructores y los asistentes, los de niveles más bajos en el escalafón, constituyen sólo la mitad del total del personal docente, mientras los niveles de titular y asociado alojan poco más de la cuarta parte de los docentes investigadores (Tabla 5), una proporción más de 5 veces superior que la de las universidades privadas. En el grupo de las universidades experimentales (Tabla 6), las diferencias con el grupo de las instituciones privadas también existen aunque son de menor magnitud que las descritas entre el grupo 


\section{Tabla 4}

\section{Categoría (escalafón) de los profesores activos de las universidades privadas venezolanas en el año 2000}

\begin{tabular}{|c|c|c|c|c|c|c|c|c|c|c|}
\hline \multirow[b]{2}{*}{ Universidades } & \multicolumn{2}{|c|}{ Titulares } & \multicolumn{2}{|c|}{ Asociados } & \multicolumn{2}{|c|}{ Agregados } & \multicolumn{2}{|c|}{ Asistentes } & \multicolumn{2}{|c|}{ Instructores } \\
\hline & $\mathbf{N}^{\circ}$ & $\%$ & $\mathbf{N}^{\circ}$ & $\%$ & $\mathbf{N}^{\circ}$ & $\%$ & $\mathbf{N}^{\circ}$ & $\%$ & $\mathbf{N}^{\circ}$ & $\%$ \\
\hline USM & 93 & 6,85 & 42 & 3,09 & 113 & 8,32 & 212 & 15,61 & 898 & 66,13 \\
\hline UCAB* & 41 & 3,57 & 47 & 4,10 & 148 & 12,90 & 503 & 43,85 & 408 & 35,58 \\
\hline UNIMET & 76 & 15,90 & 98 & 20,50 & 136 & 28,45 & 135 & 28,24 & 33 & 6,90 \\
\hline URU** & 11 & 13,75 & 12 & 15,00 & 17 & 21,25 & 26 & 32,50 & 14 & 17,50 \\
\hline UNITEC & 22 & 13,92 & 1 & 0,63 & 16 & 10,13 & 25 & 15,82 & 94 & 59,49 \\
\hline UCAT* & 2 & 0,70 & 9 & 3,19 & 22 & 7,80 & 46 & 16,31 & 199 & 70,57 \\
\hline UJMV* & 10 & 3,42 & 9 & 3,08 & 28 & 9,59 & 118 & 40,41 & 127 & 45,04 \\
\hline UNICA* & 12 & 8,11 & 0 & 0,00 & 11 & 7,43 & 12 & 8,11 & 113 & 76,35 \\
\hline UBA & SI & SI & SI & SI & SI & SI & SI & SI & SI & SI \\
\hline UGMA & 16 & 1,38 & 9 & 0,78 & 27 & 2,33 & 224 & 19,29 & 885 & 76,23 \\
\hline UFT* & 18 & 1,94 & 35 & 3,78 & 160 & 17,28 & 427 & 46,11 & 286 & 30,89 \\
\hline UNE & SI & SI & SI & SI & SI & SI & SI & SI & SI & SI \\
\hline UNY & 15 & 5,23 & 13 & 4,53 & 22 & 7,67 & 65 & 22,65 & 172 & 59,93 \\
\hline URBE & 35 & 6,85 & 40 & 7,83 & 120 & 23,48 & 178 & 34,83 & 138 & 27,01 \\
\hline UVM $^{*}$ & 2 & 2,81 & 1 & 1,41 & 6 & 8,45 & 33 & 46,48 & 29 & 40,85 \\
\hline UAH & 8 & 5,37 & 11 & 7,38 & 23 & 15,44 & 31 & 20,81 & 76 & 51,01 \\
\hline UJAP** & 24 & 20,00 & 1 & 0,83 & 2 & 1,67 & 6 & 5,00 & 87 & 72,50 \\
\hline Total $^{*}$ & 385 & 5,37 & 328 & 4,57 & 851 & 11,87 & 2.041 & 28,48 & 3559 & 49,67 \\
\hline
\end{tabular}

Fuente: OPSU (2001).

SI: Sin información. * * SI en menos del $10 \%$ y ** SI entre el 50 y $60 \%$ de los casos.

de las universidades autónomas y la $\mathrm{Si}$ món Bolívar y el de las universidades privadas. Las universidades experimentales triplican a las universidades privadas en la proporción de profesores en los dos más elevados niveles del escalafón. La proporción de Profesores en los dos niveles más bajos del escalafón alcanza casi el 70 por ciento de los docentes (Tabla 6 ).
Sólo dos universidades privadas, la Metropolitana y la Tecnológica del Centro tienen un porcentaje de profesores titulares similar al del grupo de las universidades autónomas más la Simón Bolívar. La UNIMET, incluso, mantiene porcentajes elevados en las categorías de asociado y agregado y sus docentes instructores y asistentes sumados escasamente supe- 


\section{Tabla 5}

Categoría (escalafón) de los profesores activos del grupo de seis universidades oficiales venezolanas integrado por las cinco universidades autónomas y la Universidad Simón Bolívar en el año 2000

\begin{tabular}{lcccccccccc}
\hline & \multicolumn{1}{c}{ Titulares } & \multicolumn{1}{c}{ Asociados } & \multicolumn{1}{c}{ Agregados } & \multicolumn{2}{c}{ Asistentes } & \multicolumn{2}{c}{ Instructores } \\
\hline Universidades & $\mathbf{N}^{\circ}$ & \multicolumn{1}{c}{$\%$} & \multicolumn{1}{c}{$\mathbf{N}^{\circ}$} & $\%$ & \multicolumn{1}{c}{$\mathbf{N}^{\circ}$} & $\%$ & \multicolumn{1}{c}{$\mathbf{N}^{\circ}$} & $\%$ & $\mathbf{N}^{\circ}$ & $\%$ \\
\hline UCV & 322 & 7,40 & 452 & 10,07 & 752 & 16,75 & 1.301 & 29,00 & 1.662 & 37,02 \\
ULA & 451 & 24,29 & 366 & 19,71 & 500 & 26,93 & 685 & 36,89 & 664 & 35,76 \\
LUZ & 572 & 18,82 & 538 & 17,70 & 854 & 28,09 & 489 & 16,09 & 581 & 19,11 \\
UC $^{*}$ & 256 & 12,09 & 273 & 12,89 & 383 & 18,08 & 348 & 16,43 & 504 & 23,80 \\
UDO $^{*}$ & 159 & 7,42 & 236 & 11,01 & 341 & 15,91 & 347 & 16,19 & 1.060 & 49,46 \\
USB $^{* *}$ & 154 & 28,31 & 165 & 30,33 & 172 & 31,62 & 53 & 9,74 & 0 & 0,00 \\
Total $^{*}$ & 1.914 & 13,07 & 2.030 & 13,86 & 3.002 & 20,50 & 3.223 & 22,01 & 4.471 & 30,53 \\
\hline
\end{tabular}

Fuente: OPSU (2001).

Cálculos propios.

*Sin información $5 \%$ o menos y ${ }^{* *} \sin$ información en más del $50 \%$ de los casos.

\section{Tabla 6}

Categoría (escalafón) de los profesores activos del grupo de once universidades oficiales experimentales en el año 2000

\begin{tabular}{|c|c|c|c|c|c|c|c|c|c|c|}
\hline \multirow[b]{2}{*}{ Universidades } & \multicolumn{2}{|c|}{ Titulares } & \multicolumn{2}{|c|}{ Asociados } & \multicolumn{2}{|c|}{ Agregados } & \multicolumn{2}{|c|}{ Asistentes } & \multicolumn{2}{|c|}{ Instructores } \\
\hline & No. & $\%$ & No. & $\%$ & No. & $\%$ & No. & $\%$ & No. & $\%$ \\
\hline $\mathrm{UCLA}^{*}$ & 35 & 3,18 & 110 & 9,98 & 229 & 20,78 & 465 & 42,20 & 235 & 21,32 \\
\hline UNESR & 25 & 2,02 & 0 & 3,96 & 195 & 15,75 & 464 & 37,48 & 505 & 40,79 \\
\hline UNFT* & 33 & 15,60 & 35 & 15,98 & 72 & 32,88 & 101 & 46,12 & 78 & 35,62 \\
\hline UNELLEZ & 46 & 6,55 & 96 & 13,68 & 125 & 17,81 & 195 & 27,78 & 240 & 34,19 \\
\hline UNA & 1 & 0,18 & $t$ & 1,29 & 54 & 9,94 & 133 & 24,49 & 348 & 64,09 \\
\hline UTVIULA & 22 & 8,27 & 23 & 8,65 & 47 & 17,67 & 104 & 39,10 & 70 & 26,32 \\
\hline UNEFM* & 60 & 21,05 & 54 & 18,95 & 73 & 25,61 & 94 & 32,98 & 4 & 1,40 \\
\hline JNEG & 2 & 0,64 & 13 & 4,14 & 96 & 30,57 & 144 & 45,86 & 59 & 18,79 \\
\hline UNERMB & 25 & 5,26 & 72 & 15,16 & 151 & 31,79 & 140 & 29,47 & 74 & 15,58 \\
\hline UPEL & 249 & 7,58 & 240 & 7,31 & 252 & 7,68 & 286 & 8,71 & 2.256 & 68,72 \\
\hline UNEXPO* & 75 & 11,42 & 124 & 18,87 & 132 & 20,09 & 211 & 32,12 & 115 & 17,50 \\
\hline Total $^{*}$ & 573 & 6,26 & 823 & 9,00 & 1.426 & 15,59 & 2.337 & 25,56 & 3.984 & 43,57 \\
\hline
\end{tabular}

Fuente: OPSU (2001).

Cálculos propios.

* Sin información en menos del 10\% y ** sin información entre el 50 y 60\% de los casos. 
ran un tercio del total de sus profesores. La Universidad Rafael Urdaneta presenta, en este aspecto, un perfil muy similar al del grupo que reúne a las universidades autónomas y a la Simón Bolívar, pero presenta una cifra de docentes sin información del 54,8 por ciento. Otra institución privada con una cifra similar de profesores sin información respecto a su escalafón fue la José Antonio Páez. De la Bicentenaria de Aragua y de la Nueva Esparta no se obtuvo ninguna información en este aspecto (Tabla 4).

En el caso de las universidades experimentales, la Rómulo Gallegos y la Francisco de Miranda presentaron cifras mayores al 50 por ciento de profesores sin información (Tabla 6); otro tanto ocurrió con la Universidad Simón Bolívar (Tabla 5).

\subsection{Relación Alumno/Profesor}

La relación alumno/profesor de los tres grupos de universidades estudiadas se presenta en la Tabla 7. Las universidades privadas presentan una relación del doble de alumnos por profesor tiempo completo equivalente, al existente en las universidades oficiales. La comparación se hace más desventajosa a las instituciones privadas si se efectúa contra el grupo de las universidades autónomas más la Simón Bolívar, cuya relación alumno/profesor es el 43 por ciento de la existente en las universidades privadas. Si la comparación se efectúa entre las universidades privadas y las experimentales, el resultado sigue favoreciendo a las universidades oficiales frente a las privadas, pues la relación alumno/profesor de las universidades experimentales es el $66,7 \%$ de la relación existente en las universidades privadas. La Tabla 7 también muestra que el número de estudiantes totales de las universidades oficiales es casi 3,5 veces mayor que el de las universidades privadas.

\section{Tabla 7}

\section{Comparación de la matrícula, número de profesores tiempo completo equivalente y relación de estudiantes/profesor tiempo completo equivalente en universidades privadas y universidades oficiales para el año 2000}

\begin{tabular}{cccc}
\hline $\begin{array}{c}\text { Grupos de Universidades } \\
\text { Privadas y Oficiales }\end{array}$ & $\begin{array}{c}\mathbf{N}^{\circ} \text { de estudiantes } \\
\text { totales (matrícula) }\end{array}$ & $\begin{array}{c}\mathbf{N}^{\circ} \text { Profesores } \\
\text { Tiempo Completo } \\
\text { Equivalente }\end{array}$ & $\begin{array}{c}\text { Relación estudiante/ } \\
\text { prof Tiempo completo } \\
\text { Equivalente }\end{array}$ \\
\hline $\begin{array}{c}\text { Privadas } \\
\text { Autónomas } \\
+ \text { USB }\end{array}$ & 111.748 & $2.996,75$ & 37,29 \\
$\begin{array}{c}\text { Experimentales } \\
\text { - USB }\end{array}$ & 176.142 & 12.833 & 16,02 \\
$\begin{array}{c}\text { Total de Universidades } \\
\text { Oficiales }\end{array}$ & 381.702 & 7079,50 & 24,88 \\
\hline
\end{tabular}

Fuente: OPSU (2001).

Cálculos propios. 


\subsection{Nivel Científico del personal docente}

La comparación, entre los tres grupos de universidades, del porcentaje de sus profesores con estudios de postgrado, demuestra que las universidades oficiales tienen una mayor proporción de profesores con postgrado que las instituciones privadas (Tabla 8), siendo favorecido el grupo de las universidades experimentales, cuya proporción de docentes con postgrado fue superior en un $19 \%$ a la proporción mostrada por el grupo de las universidades autónomas más la Universidad Simón Bolívar (cálculo obtenido de las cifras de la Tabla 8). De la misma tabla también se extrae que el número de docentes de las universidades oficiales es 2,6 veces mayor que el de las universidades privadas.

En la Tabla 9 se compara el número de docentes de las universidades del país reconocidos como investigadores por el Programa de Promoción del Investigador, así como su proporción respecto al total de profesores contratados a dedicación exclusiva más aquéllos contratados a tiempo completo. La proporción de investigadores es mayor en el grupo de universidades autónomas más la Simón Bolívar que en el resto de los grupos estudiados y es mayor en las universidades experimentales que en las instituciones privadas (Tabla 9). Así, las universidades autónomas más la USB tienen un porcentaje de docentes reconocidos como investigadores 21 veces mayor que el de las universidades privadas y 6 veces mayor que el grupo de las universidades experimentales (cifras calculadas de la Tabla 9). Éstas, por su parte, tienen un porcentaje de investigadores tres veces y medio mayor que el de las universidades privadas (Tabla 9). Sólo 6 profesores de las universidades privadas están reconocidos como investigadores contra 1.512 investigadores reconocidos de las univer-

\section{Tabla 8}

Comparación del número y proporción de profesores con título de postgrado en universidades privadas y universidades oficiales para el año 2000

\begin{tabular}{cccc}
\hline $\begin{array}{c}\text { Grupos de Universidades } \\
\text { Privadas y Oficiales }\end{array}$ & $\mathbf{N}^{\circ}$ de docentes & $\begin{array}{c}\mathbf{N}^{\circ} \text { Profesores } \\
\text { con postgrado }\end{array}$ & $\begin{array}{c}\text { \% de docentes } \\
\text { con postgrado }\end{array}$ \\
\hline Privadas & 8.067 & 2.512 & 31,13 \\
Autónomas & 13.253 & 5.351 & 40,37 \\
+ USB & & & 47,90 \\
$\begin{array}{c}\text { Experimentales } \\
\text { - USB }\end{array}$ & 7.552 & 3.618 & \\
$\begin{array}{c}\text { Total de Universidades } \\
\text { Oficiales }\end{array}$ & 20.805 & 8.969 & 43,10 \\
\hline
\end{tabular}

Fuente: OPSU (2001).

Cálculos propios. 


\section{Tabla 9}

\section{Comparación del número y proporción de profesores reconocidos por el Programa de Promoción del Investigador en universidades privadas y universidades oficiales para el año 2000}

\begin{tabular}{cccc}
\hline $\begin{array}{c}\text { Grupos de Universidades } \\
\text { Privadas y Oficiales }\end{array}$ & $\begin{array}{c}\mathbf{N}^{\circ} \text { Profesores } \\
\text { DE + TC }\end{array}$ & $\begin{array}{c}\mathbf{N}^{\circ} \text { Profesores } \\
\text { reconocidos por el PPI }\end{array}$ & $\begin{array}{c}\text { Porcentaje de } \\
\text { profesores DE + TC } \\
\text { en el PPI }\end{array}$ \\
\hline $\begin{array}{c}\text { Privadas } \\
\text { Autónomas } \\
\quad+\text { USB }\end{array}$ & 1.006 & 6 & 0,60 \\
$\begin{array}{c}\text { Experimentales } \\
\text { - USB }\end{array}$ & 11.118 & 1.393 & 12,53 \\
$\begin{array}{c}\text { Total de Universidades } \\
\text { Oficiales }\end{array}$ & 16.743 & 119 & 2,12 \\
\hline
\end{tabular}

Fuente: OPSU (2001).

Cálculos propios.

sidades oficiales: 1.393 del grupo de las autónomas y la USB y 119 en las universidades experimentales.

La Tabla 9 también muestra que la suma de profesores contratados en las dos dedicaciones mayores (dedicación exclusiva + tiempo completo) es mayor en el caso de las universidades oficiales que en las universidades privadas, situación ya presentada previamente al iniciar la presentación de estos resultados ( $\mathrm{Ta}$ blas 1,2 y 3 ).

\subsection{Oferta de programas de postgrado}

El desempeño de las universidades estudiadas en la docencia de postgrado se puede observar en las Tablas siguientes, en los que se muestra el número y proporción de programas de postgrado clasificados por tipo según grado de estudios, dictados actualmente por las universidades de cada uno de los tres grupos en estudio (Tabla 10) y el número y proporción de postgrados acreditados por el CNU, según grado al que conducen, dictados igualmente por las universidades venezolanas de los tres grupos estudiados (Tabla 11).

De los 1.566 programas de postgrado existentes para el 30 de marzo de 2003, sólo 44 no son dictados por las universidades; de éstos últimos, más de la mitad (26), corresponden al Instituto Venezolano de Investigaciones Científicas (IVIC), de manera que las universidades concentran el 97,19 por ciento del total de los postgrados del país y el IVIC el 1,66\%. De los postgrados dictados por las universidades, más de la mitad corresponden a especializaciones y menos del 10 por ciento a doctorados (Tabla 10); sólo una cuarta parte de los programas de postgrado han sido acreditados (Tabla 11). De los programas acreditados, menos del 10 por ciento corresponde a doctorados, mientras el 90 por ciento res- 


\section{Tabla 10}

Número y proporción de programas de postgrado ofrecidos por las universidades venezolanas de los tres grupos estudiados clasificados según el tipo de grado al que conducen. Año 2003

\begin{tabular}{|c|c|c|c|c|c|c|c|c|}
\hline \multirow[b]{2}{*}{ Grupos de Universidades } & \multicolumn{2}{|c|}{ Especialización } & \multicolumn{2}{|c|}{ Maestrías } & \multicolumn{2}{|c|}{ Doctorados } & \multicolumn{2}{|c|}{ Totales } \\
\hline & $\mathbf{N}^{\circ}$ & $\%$ & $\mathbf{N}^{\circ}$ & $\%$ & $\mathbf{N}^{\circ}$ & $\%$ & $\mathbf{N}^{\circ}$ & $\%$ \\
\hline Privadas & 178 & 11,70 & 116 & 7,62 & 14 & 0,92 & 308 & 20,24 \\
\hline $\begin{array}{c}\text { Autónomas } \\
+ \text { USB }\end{array}$ & 461 & 30,29 & 345 & 22,67 & 87 & 5,72 & 893 & 58,67 \\
\hline $\begin{array}{c}\text { Experimentales } \\
\text { - USB }\end{array}$ & 150 & 9,86 & 163 & 10,71 & 8 & 0,53 & 321 & 21,09 \\
\hline Totales & 789 & 51,84 & 624 & 41,00 & 109 & 7,16 & 1.522 & 100,00 \\
\hline
\end{tabular}

Fuente: Consejo Consultivo Nacional de Estudios de Postgrado, CNU, marzo 2003.

Cálculos propios.

Tabla 11

Número y proporción de programas de postgrados acreditados dictados por las universidades venezolanas de los tres grupos estudiados clasificados por el tipo de grado a que conducen. Año 2003

\begin{tabular}{|c|c|c|c|c|c|c|c|c|}
\hline \multirow[b]{2}{*}{ Grupos de Universidades } & \multicolumn{2}{|c|}{ Especialización } & \multicolumn{2}{|c|}{ Maestrías } & \multicolumn{2}{|c|}{ Doctorados } & \multicolumn{2}{|c|}{ Totales } \\
\hline & $\mathbf{N}^{\circ}$ & $\%$ & $\mathbf{N}^{\circ}$ & $\%$ & $\mathbf{N}^{\circ}$ & $\%$ & $\mathbf{N}^{\circ}$ & $\%$ \\
\hline Privadas & 22 & 5,80 & 10 & 2,64 & 0 & 0 & 32 & 8,44 \\
\hline \multicolumn{9}{|l|}{ Autónomas } \\
\hline+ USB & 120 & 31,66 & 145 & 38,26 & 35 & 9,23 & 300 & 79,16 \\
\hline \multicolumn{9}{|l|}{ Experimentales } \\
\hline - USB & 26 & 6,86 & 20 & 5,28 & 1 & 0,26 & 47 & 12,40 \\
\hline Totales & 168 & 44,33 & 175 & 46,17 & 36 & 9,50 & 379 & 100,00 \\
\hline
\end{tabular}

Postgrados acreditados/postgrados totales: $25,23 \%$.

Fuente: Consejo Consultivo Nacional de Estudios de Postgrado, CNU, marzo 2003.

Cálculos propios.

tante corresponde casi por igual a especializaciones y maestrías (Tabla 11).

Del total de los programas de postgrado existentes, las cuatro quintas partes son dictados por las universidades oficiales y sólo una quinta parte por las instituciones privadas (Tabla 10). El grupo de las universidades oficiales autónomas y la Simón Bolívar prácticamente tienen el 60 por ciento de todos los postgrados; el número restante se reparte por igual entre el grupo de las universidades experimentales sin la Simón Bolívar y las universidades privadas (Tabla 10). Esta misma tabla nos revela la supremacía del grupo de las universidades autónomas y 
la USB en cada uno de los tipos de postgrado: especializaciones, maestrías y doctorados, en los cuales duplica y hasta decuplica a los otros dos grupos de universidades. La Tabla 10 no muestra la existencia de diferencias importantes entre el grupo de las universidades privadas y el grupo de las experimentales sin la Universidad Simón Bolívar en cuanto a número o proporción de los postgrados ofrecidos, ni al tipo de los mismos.

En cuanto a los postgrados acreditados, nuevamente la inmensa mayoría de los mismos, más del 90 por ciento, pertenece a las universidades oficiales ( $\mathrm{Ta}$ bla 11) y, dentro de éstas, al grupo de las universidades autónomas más la Simón Bolívar, el cual tiene un número de especializaciones, maestrías y doctorados acreditados 4,6, 7,3 y 35 veces mayor, respectivamente, que el grupo de las universidades experimentales sin la Simón Bolívar (datos calculados de la Tabla 11). Las universidades experimentales, por su parte, aventajaron discretamente a las privadas en el número y proporción de especializaciones, maestrías y doctorados acreditados.

\section{Discusión, conclusiones y propuestas}

De los resultados mostrados, con relación a la dedicación de los profesores de los tres grupos de universidades estudiados, se desprende que la enseñanza de pregrado y de postgrado de las universidades privadas descansa totalmente en profesores a tiempo convencional, es decir en docentes que están contratados únicamente para el dictado de horas de clases. Casi el 80 por ciento de los docen- tes se encuentran en esta categoría (Tabla 1) y del casi 15 por ciento contratado a dedicación exclusiva y tiempo completo hay que restar aquellos docentes que ejercen la administración académica: autoridades, directores, jefaturas de programas y otros, quienes generalmente no tienen actividades académicas a su cargo o las tienen en forma muy reducida.

Más de la mitad de las universidades privadas no tienen personal académico dedicado exclusivamente a la institución y sólo dos de ellas tienen más de 30 por ciento de su personal docente contratado a tiempo completo (Tabla 1), lo que significa que una serie de actividades académicas, distintas del dictado de clases de pregrado o postgrado, se encuentran severamente limitadas en estas instituciones universitarias. No habría tiempo contratado para la realización consuetudinaria de actividades de investigación, ni para el desarrollo de labores de extensión universitaria.

Una imagen en espejo se produce en los dos grupos de universidades oficiales, sin importar si se trata de las universidades autónomas 0 de las experimentales. En éstas, alrededor del 20 por ciento de los docentes está contratado a tiempo convencional, mientras la gran mayoría, casi el 70 por ciento (Tablas 2 y 3) están contratados a tiempo completo y dedicación exclusiva, dedicaciones que permiten no sólo el dictado de clases de pregrado, sino las actividades de consulta con los estudiantes de pregrado, la realización permanente de labores de investigación y extensión y la participación en la docencia y formación de postgrado, actividades todas que estarían severamente limitadas en las universidades priva- 
das, al estar desprovistas de personal académico de planta.

Habría que establecer un alerta con relación a aquellas universidades oficiales en las que el personal contratado a tiempo convencional constituye la mayor parte de su planta docente: la Pedagógica Experimental Libertador y la Rómulo Gallegos (Tabla 3), así como las cuatro instituciones de más reciente creación: la Universidad Sur del Lago, la de la Fuerza Armada Nacional, la Marítima del Caribe y la de Yaracuy. De mantenerse esta situación se verían fuertemente limitadas en el desarrollo de sus actividades académicas, más allá del dictado de clases de pregrado y postgrado.

Las limitaciones académicas señaladas para las universidades privadas se ratifican y profundizan, cuando se procede al análisis de los resultados obtenidos en este estudio respecto a la calificación académica de los docentes de acuerdo a su categoría en el escalafón universitario. De la comparación entre los tres grupos de universidades, nuevamente las instituciones privadas quedan en desventaja, pues casi el 80 por ciento de sus profesores está ubicado en las dos categorías más bajas del escalafón: la de instructor y la de profesor asistente (Tabla 4), lo que significa que se trata del personal con menor preparación y experiencia, personal docente y de investigación supuestamente en formación, que le hace imposible abordar con toda propiedad actividades académicas como la producción de conocimientos y la docencia de postgrado y le limita el desarrollo adecuado de la actividad docente de pregrado y la realización de labores de extensión universitaria.
La situación deficitaria en cuanto a la calificación académica se agrava si tomamos en cuenta que el personal preparado o formado es solamente un 10 por ciento del total, según los datos obtenidos en este estudio y mostrados en la misma Tabla 4; esto limitaría la capacidad institucional para preparar y formar a la planta docente de instructores y asistentes, quienes deberían estar en formación como docentes e investigadores, lo cual realmente no es el caso de las universidades privadas. Esta situación contrasta con los resultados obtenidos en ambos grupos de las universidades oficiales (Tablas 5 y 6), especialmente cuando se comparan los datos con los del grupo de universidades que incluye a las autónomas y la Simón Bolívar (Tablas 5), el cual aventaja con claridad en estos aspectos al grupo de las universidades oficiales experimentales (Tabla 6).

En el grupo de las universidades autónomas más la Simón Bolívar existe una relación aproximada entre el personal formado, en principio el calificado en los dos más altos niveles del escalafón, y el personal en formación de 1:2, mientras en las universidades privadas la proporción es de 1:8. Por su parte, el personal en formación en las universidades oficiales es realmente un personal en período de formación con tutores designados y un programa académico a ser cumplido dentro del lapso establecido para ello. Éste no es el caso en las universidades privadas, en las que este personal no está sometido a ningún programa ni a supervisión de sus actividades académicas. Se trata, además de un personal de alta rotación, recién graduado, sin ningún tipo de experiencia. 
En el caso de las universidades privadas, además, la presencia de profesores clasificados en posiciones elevadas del escalafón no obedece a la existencia de una carrera académica en estas instituciones, que sea la responsable del proceso de formación de esos docentes y que haya permitido e impulsado el ascenso de éstos dentro de las instituciones. Se trata en realidad de profesores ya formados en las universidades oficiales, quienes pasan a prestar servicios en el sector educativo privado luego de hacer efectivas sus jubilaciones en el sector público. De las universidades privadas, solamente la UNIMET y en menor grado la Universidad Tecnológica del Centro no estarían tan limitadas académicamente como consecuencia de la categoría de sus docentes, aunque las seguirían teniendo como consecuencia de la baja dedicación de los mismos.

El estudio de la relación alumno/profesor mostrada en la Tabla 7 demuestra que la docencia de pregrado está cuantitativamente mejor atendida en los dos grupos de universidades oficiales estudiados, que en el grupo de las universidades privadas. Si tomamos en cuenta también los resultados ya discutidos sobre la dedicación y formación de los docentes en los tres grupos de instituciones investigadas, podemos afirmar sin temor a equivocarnos que la docencia de pregrado también está cualitativamente mejor atendida en las universidades oficiales que en las privadas, lo que confirma el análisis y conclusiones que venimos sosteniendo como producto del estudio de los datos obtenidos.

Cuando nos detenemos en el análisis de las diferencias de formación de los docentes de los tres grupos de universidades, encontramos que los profesores con postgrado son proporcionalmente más numerosos en los dos grupos de universidades oficiales que en el grupo de universidades privadas. Esto significa que los docentes de las universidades oficiales están más preparados para el ejercicio de todas las actividades académicas, incluida la docencia de pregrado. Además, se confirmarían las limitaciones de las universidades privadas para la realización de actividades de postgrado y de investigación.

De nuevo podemos señalar que la presencia de formación de postgrado en un tercio de los docentes de las universidades privadas (Tabla 8) no es una consecuencia del desarrollo de un esfuerzo en este sentido por estas instituciones, sino es producto de la contratación de profesores con postgrado provenientes de las universidades oficiales. Una parte de éstos está dedicado a la administración académica y a las labores del gobierno universitario, lo que lo restringe de participar en el desarrollo de las actividades académicas.

Los datos de la Tabla 9 completan la evaluación académica de los profesores de las universidades privadas y su comparación con los docentes de las universidades oficiales, divididas en este trabajo en dos grupos diferentes. Las universidades privadas prácticamente no poseen investigadores reconocidos por el PPI, pues solamente 6 profesores de un total de más de un millar está reconocido como investigador, mientras la cifra de investigadores reconocidos en las universidades oficiales es mucho mayor, incluso en el grupo de universidades experi- 
mentales sin la presencia de la Simón Bolívar, en las que existe más de un centenar de investigadores (Tabla 9) de un total de más de 5.600 docentes. Si comparamos los porcentajes encontraremos que este grupo de universidades experimentales, cuya proporción de investigadores reconocidos por el PPI es inaceptablemente bajo, tiene un porcentaje de 3,5 veces superior de investigadores reconocidos, que las universidades privadas.

La comparación con la proporción de investigadores reconocidos del grupo de universidades autónomas más la Simón Bolívar, aunque la cifra también se nos antoja inaceptablemente baja $(12,53 \%)$, lo que deja a los otros dos grupos muy mal parados. Este grupo de universidades oficiales tiene 21 veces más investigadores que las universidades privadas y 6 veces más investigadores reconocidos que las universidades experimentales (cálculos a partir de los datos de la tabla 8).

Estos datos claramente significan que en las universidades privadas no se hace investigación científica o ésta, al menos, es muy reducida o marginal con respecto a la docencia de pregrado. Estos últimos datos nos dicen también que las instituciones universitarias privadas están limitadas para el desarrollo del nivel de postgrado, principalmente para el desarrollo de postgrados académicos, entendidos éstos como aquéllos que conducen a los grados de magíster y doctor, es decir los postgrados que forman investigadores. No dispondrían tampoco de personal capacitado para la realización de actividades de extensión de cierta complejidad, como las que se derivan de convenios con organismos públicos, en los que la realización de estudios e investigaciones, preceden a las acciones.

La limitación de las universidades privadas en el área de postgrado se evidencia con claridad de los datos organizados en las dos últimas tablas. Las universidades públicas dictan la inmensa mayoría del total de los cursos de postgrado existentes en el país y dentro de ellas, las autónomas más la Simón Bolívar dictan casi el 60 por ciento de los mismos (Tabla 10), distribuyéndose el resto por igual entre las universidades experimentales y las privadas. La menor realización de especializaciones, maestrías y doctorados, por parte de las universidades privadas, concuerda completamente con todos los datos ya señalados y discutidos. Esa limitación es mucho mayor en los doctorados que en las maestrías, donde la proporción es 6,2 veces superior en el grupo de las universidades oficiales autónomas más la Simón Bolívar que en el de las universidades privadas. El grupo de las universidades experimentales sin la Simón Bolívar, por su parte, desarrolla actividades de postgrado muy similares al de las universidades privadas.

Cuando la comparación entre las universidades se efectúa con relación al desarrollo de postgrados acreditados, las diferencias son todavía mayores en desmedro de las universidades privadas. Entendiendo que solamente la cuarta parte de los postgrados dictados se encuentran acreditados, del total de estos últimos, casi el 80 por ciento pertenece al grupo de las universidades autónomas más la USB, mientras el $12 \%$ pertenece al grupo de las universidades experimentales $y$ sólo el $8 \%$ es desarrollado por las universidades privadas (Tabla 11). Las diferen- 
cias son notorias en los tres tipos de postgrado, según la naturaleza del grado conferido, en los cuales las universidades oficiales autónomas más la USB demuestran claramente su primacía.

Estos resultados no son de extrañar pues son una clara consecuencia de los datos presentados y ya discutidos anteriormente. Así, si las instituciones privadas no poseen personal de planta en cantidad suficiente, sino solamente docentes a tiempo convencional; si además carecen de personal preparado en número suficiente, lo cual se evidencia porque la inmensa mayoría de sus docentes están ubicados en los primeros pasos del escalafón y menos de un tercio de los mismos tienen estudios de postgrado, y si no tienen personal reconocido como investigador, es imposible pensar que puedan desarrollar programas de postgrado en el $\mathrm{ni}$ vel de maestrías y doctorados como lo realizan las universidades oficiales, fundamentalmente el grupo de las autónomas más la USB.

Es claro en la investigación realizada que la universidad privada venezolana tiene limitaciones superables en su actividad docente de pregrado, mientras que sus deficiencias en el nivel del postgrado y en investigación científica tienen una base de carácter más estructural y, por lo tanto, no igualmente superables; por lo menos no en el sentido de poderla llevar hasta el desarrollo cuantitativo y cualitativo que podrían alcanzar en la universidad oficial. Así, la limitante que significa la falta de una planta docente contratada a dedicación exclusiva o tiempo completo puede ser resuelta de inmediato mediante un incremento en la contratación de profesores a estas dedicaciones, sin que esta medida signifique que la proporción de docentes con contrataciones máximas deba ser similar a la existente en la universidad oficial. Recordemos que la universidad privada venezolana se autofinancia con los recursos provenientes del pago de las mensualidades y los gastos de inscripción efectuados por sus estudiantes de pregrado principalmente; por lo tanto, llevar la proporción de profesores a dedicación exclusiva y tiempo completo a los niveles existentes en la universidad oficial puede encarecer tanto el costo de los estudios, que la hagan imposible de mantenerse.

Una proporción menor de profesores de planta, sí, pero una cierta proporción, contratada a dedicación exclusiva, homogéneamente distribuida entre todos los programas académicos, distinta de la planta docente de autoridades, directivos y administradores de la academia, que permita la realización de las consultas docentes de los estudiantes en una mayor proporción y que deje tiempo suficiente para la formación del docente como investigador y para el desarrollo de las otras actividades académicas. Una medida de esta naturaleza, instaurada mediante un mandato de carácter legal, con seguridad contribuiría a mejorar la calidad de la educación universitaria privada. La cifra a requerir siempre podrá ser discutida pero, basado en las contrataciones actuales de las instituciones privadas mostradas en la Tabla 1 y las existentes en las universidades oficiales (Tablas $2 y$ 3 ), propondría que un 33 por ciento de los docentes sean contratados a dedicación exclusiva, sin tomar en cuenta para el cálculo del porcentaje los docentes en funciones de autoridades, ni los directivos, ni 
los administradores docentes. Esta cifra no constituiría una carga que, desde el punto de vista presupuestario y financiero, no pudiera ser asumida por las universidades privadas sin ningún peligro. Estos docentes dispondrían de tiempo para el desarrollo de actividades de investigación y extensión. Las limitaciones para el desarrollo de los postgrados, sobretodo los del nivel de maestría y de doctorado, dirigidos a formar investigadores, en cambio, son de un carácter más ligado a las limitaciones estructurales de la universidad privada venezolana dadas por el origen de su financiamiento. Estos postgrados tienen mucho mayores necesidades de personal altamente capacitado: la existencia de investigadores formados y con una producción permanente e importante de conocimientos, que puedan actuar como tutores, supervisores y consultores y permitan una fácil incorporación del estudiante al ambiente científico y al trabajo creativo formador.

No se trata de profesionales que puedan ser contratados individualmente; generalmente son equipos de trabajo que han requerido de años para su formación y su constitución. Requieren además de bibliotecas especializadas bien surtidas, de laboratorios costosamente equipados y de trabajos de campo laboriosos y costosos, que hacen imposible su realización consuetudinaria si no existe financiamiento suficiente y permanente, el cual, en Venezuela, sólo tiene como fuente al Estado venezolano.

La limitación anterior es también válida para el desarrollo de la investigación científica y humanística o mejor definida, para la creación intelectual en todas sus formas, actividad fundamental de la universidad de hoy (Fuenmayor, 2001), pues la misma no podría ser costeada con la fuente de financiamiento de la universidad privada venezolana, como ya lo hemos señalado. Sin embargo, no debería haber impedimento para que el personal académico a dedicación exclusiva utilice por lo menos una quinta parte de su tiempo, un día a la semana por ejemplo, en la realización de actividades de investigación dentro de una unidad de investigación de una universidad oficial, a la cual sería formalmente adscrito.

Esta unidad se vería favorecida al disponer de un profesional adicional formado o en formación, como investigador trabajando permanentemente con el equipo regular de investigadores, lo que debería incrementar la producción científica del grupo. La institución privada se favorecería al disponer para su profesorado de planta de un sitio de trabajo equipado y con investigadores formados, cuya organización y funcionamiento no tendría que financiar. Sólo financiaría los gastos consumibles del trabajo de investigación del docente de la institución adscrito formalmente a la unidad. Se beneficiaría el docente y con ello su institución, pues sus labores académicas darían un salto cualitativo y estaría en capacidad de asumir la docencia de pregrado en la mejor forma posible. Estaría, además, preparado para la realización de docencia de postgrado en su disciplina y otras afines. La proposición anterior debe instrumentarse y generalizarse su implementación mediante un mandato legal en la legislación próxima a ser aprobada sobre la educación superior.

La extensión universitaria en las instituciones privadas no ha sido objeto 
de nuestro estudio, aunque sí lo es de nuestra preocupación. La dedicación actual a tiempo convencional de los docentes de las universidades privadas limita grandemente la realización de estas actividades. Al hacer surgir un personal de planta a dedicación exclusiva, tal y como lo hemos propuesto anteriormente, se crean las condiciones para la realización de las actividades de extensión. Las mismas deberían ser articuladas con la docencia normal de pregrado, a fin de impulsarlas en forma significativa dentro de las actividades ordinarias de las universidades privadas. Deberían estar dirigidas hacia la prestación de servicios a la comunidad por parte de los estudiantes, supervisados en el desarrollo de esta prestación por sus docentes; esta labor constituiría parte de las actividades académicas de formación profesional de los mismos. Recordemos que incluso en las universidades oficiales la extensión es la función universitaria de menor desarrollo.

\section{Bibliografía Citada}

Ágreda, José Ángel (1986), "Gratuidad de la Educación Superior", Exposición, Debate abierto en la Casa del Profesor Universitario, Asociación de Profesores de la Universidad Central de Venezuela, 27 de febrero, Caracas.

Asamblea Nacional Constituyente (1999), Constitución de la República Bolivariana de Venezuela. Sairam Editores S.R.L., Talleres Gráficos Arteaga, S.A., Lima, Perú.

Congreso Nacional (1992), "Ley de Universidades 1970", en: Legislación Universitaria, Universidad Central de Venezuela, Oficina Central de Asesoría Ju- rídica, ediciones del Rectorado, pp 163-212, Caracas.

Consejo Nacional de Universidades. Oficina de Planificación del Sector Universitario (2001), "Sistema de evaluación y acreditación de las universidades nacionales" (Cesar Villarroel y Comisión Técnica del Proyecto SEA) Cuadernos OPSU, $\mathrm{N}^{\circ} 3,86 \mathrm{P}$, noviembre, Caracas.

Consejo Nacional de Universidades. Oficina de Planificación del Sector Universitario (2002), Tipología de las Universidades Venezolanas (Mimeografiado) Proyecto Alma Mater, Sistema de Evaluación y Acreditación, 30 P, Noviembre, Caracas.

Fuenmayor Toro, Luis (1986), Hacia la definición de una política universitaria, Gaceta apucv/ipp, año 7, № 47 , pp 1-2, junio, Caracas.

Fuenmayor Toro, Luis (2001), "Orgánica también para la educación Superior", en: "Transformación: Urgencia de la universidad venezolana" (CNU-Secretaría Permanente, editores), pp 111-118, noviembre, Caracas.

Fuenmayor Toro, Luis (2002), "Historia, desarrollo y perspectivas del sector universitario venezolano". Cuadernos OPSU, N5, pp 1-36, enero, Caracas.

OPSU (2001), Proyecto Alma Mater. Sistema de evaluación y acreditación.

Ramírez, T.; Méndez, P. y Bravo, L. (1998), Investigación documental y bibliográfica: Recomendación para la práctica estudiantil. p 21. Editorial Panapo, Caracas.

Selltiz, C. y Jahoda, M. (1977), Los métodos de investigación en las ciencias sociales. p 22. Editorial Rialp, Madrid.

UNESCO (1997), Seminario de Educación Superior en el siglo XXI: Visión de América Latina y el Caribe. La Habana. 